\title{
Outcome of Lens-Sparing Vitrectomy for Infantile Post-Traumatic Amblyogenic Vitreous Haemorrhage: A case report
}

\author{
AO Hassan, ${ }^{1}$ ON Okonkwo, FG Adepoju, ${ }^{2}$ R Duke, ${ }_{1}$ O Oderinlo, ${ }_{1}^{1}$ F Oluyadi, ${ }^{1}$ A Ogunro, ${ }^{1}$ M Ulaikere, ${ }^{1}$ \\ A Harriman ${ }^{1}$ \\ ${ }^{1}$ Eye Foundation Retina Institute, Ijebu Imushin, Ogun State \\ ${ }^{2}$ Department of Ophthalmology, University of Ilorin Teaching Hospital, Ilorin, Kwara State
}

\begin{abstract}
A BStract
The visual outcome after bilateral lens-sparing 3-port pars plana

vitrectomy in a case of post-traumatic vitreous haemorrhage in an infant is reported. Although it is possible to perform a vitrectomy procedure via a pars plana approach in infants without compromise to the lens for the treatment of amblyogenic vitreous opacity, such as vitreous haemorrhage, the visual outcome could be poor in cases associated with trauma owing to possible structural damage to the macular, the optic nerve and other more posterior visual pathways.
\end{abstract}

Key words: ocular trauma in infants, vitreous haemorrhage, lens-sparing vitrectomy

\section{B ACKGROUND}

Vitrectomy procedure has been recommended as early as 3 to 4 weeks after the onset of a dense, infantile, vitreous haemorrhage in an attempt to avert serious complications. ${ }^{1}$ Some of the possible complications of infantile vitreous haemorrhage noted in the cases of consecutive infants include, traction retinal detachment, epiretinal membrane formation, pigmentary retinopathy, strabismus, large anisometropic myopia and occlusion amblyopia. ${ }^{1}$

Lensectomy is usually performed in infant eyes undergoing vitreoretinal surgery. However, Maguire and Trese ${ }^{2}$ have shown that in selected cases vitreoretinal surgery can be performed in infants without lens removal.

There are now several case reports of lens sparing vitrectomy performed on infants for the treatment of advanced stages of retinopathy of prematurity with tractional retinal detachment as well as vitreous opacities including vitreous haemorrhage. . $^{3,4,6}$

In paediatric eyes, when lens sparing vitrectomy is performed, lens clarity has been observed over a long period of follow up. In 85 eyes of 77 paediatric patients who underwent lens sparing vitrectomy and were observed for 10 to 55 months, 67 per cent of paediatric lenses remained clear after the surgery. ${ }^{5}$ The presence of a post-operative clear lens, therefore, improves the prognosis and visual rehabilitation, as intraoperative lens removal could further complicate the post-operative management, and necessitate aphakic correction, contact lens use or intraocular lens insertion at a later date.

Motorcycle-related accidents have been reported as the most common cause of ocular injuries in a study aimed at identifying the factors contributing to ocular trauma. It was observed that the conjunctiva, lids and cornea were the most common ocular structures affected.?

Local studies (using ultrasound scan following trauma), have reported the occurrence of posterior segment disease. ${ }^{8,9}$ In one study the youngest patient was one and half years, and the most common posterior segment lesion diagnosed was retinal detachment $(22.5 \%$ cases $)$, followed by vitreous haemorrhage $(18.3 \%){ }^{8}$

\section{CASE PRESEnTAtion}

The case of a one-year-old girl who presented with bilateral poor vision following accidental trauma, resulting in multiple head injury was reported. She was hit by a moving car and was unconscious for two weeks post injury. She had the first ophthalmology review six weeks post injury (by then she had regained full consciousness). A diagnosis of bilateral post-traumatic vitreous haemorrhage was made.

Further examination revealed that the impact of the trauma was more on the right side of the head and face, with bleeding occurring from the right nostril after the accident.

A CT scan done in the immediate post-trauma period revealed the following:
i. Occipital and fronto-parietal bone fractures
ii. Intracerebral haemorrhage
iii. Bilateral frontal lobe haemorrhagic and non haemorrhagic contusion 
iv. Extensive cerebral oedema

v. Minimal subdural haematoma

vi Multiple subcutaneous emphysema, noted in the soft tissue, outside the cranial vault, worse on the right.

\section{Ocular Examination}

Visual acuity of light perception with poor following in both eyes. Pupils were sluggish in reaction to light. The rest of the anterior segment was normal as clear lenses were observed in both eyes. There was no view of the fundus on dilated examination as a dense vitreous haemorrhage precluded fundus view in both eyes. Intraocular pressure (IOP) was within normal limits.

The digital ultrasound confirmed the presence of bilateral flat retinae and dense vitreous haemorrhage in both eyes. There was no posterior vitreous detachment. The relevance of this research to ocular trauma cases has been well documented in our local setting.,

A bilateral three port pars plana vitrectomy was performed for the removal of vitreous haemorrhage. Right eye surgery was performed on the $20^{\text {th }}$ of April 2007 and left eye on the $4^{\text {th }}$ of May ( 2 weeks interval).

\section{Surgical Method}

A 3-port vitrectomy technique with separate infusion, light pipe and vitreous cutter was used. A 21-gauge vitrectomy set and the accurus vitrectomy machine (Alcon) were used. Initial peritotomy with wet field cautery application to the bleeding vessels was done. Three sclerotomies were fashioned $2.0 \mathrm{~mm}-2.5 \mathrm{~mm}$ posterior to the limbus.

Core vitrectomy was performed with separate infusion and fiberoptic light source. Afterwards, a peripheral dissection of the vitreous base was attempted, but not aggressively done to avoid peripheral lens touch by the vitrectomy instrumentation. There was no particular effort made to induce a posterior vitreous detachment or to incise the posterior vitreous cortex.

Surgery was concluded with closure of the three sclerotomies and conjunctiva with $7 / 0$ vicryl sutures, followed by injection of subconjunctival genticin $20 \mathrm{mg}$ and betnesol $0.4 \mathrm{mg}$. eye shield was put in place.

Intraoperative findings in both eyes include: an attached retina; the presence of severe ( 3 plus) disc pallor; pigmentary macular mottling and scarring; severe arteriolar and venous narrowing and attenuation. The above findings appeared to be accentuated in the right eye,. which bore the impact of the trauma.

The patient was placed on a topical antibiotic (G. Ciloxan 4 hourly) and a steroid (G. Maxitrol 4 hourly), as well as on mydriatics (G. Mydriacyl ).These topical medications were gradually reduced and discontinued after 6-8 weeks of use.

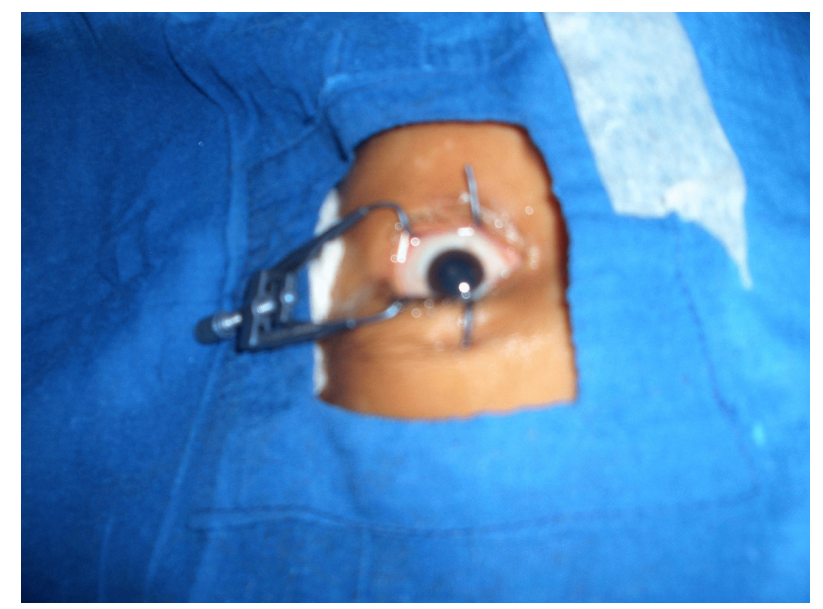

Figure 1. Left eye in the immediate post-vitrectomy period, black pupil is restored after vitreous haemorrhage removal.

\section{Results / Outcome}

Post-operative recovery was uneventful. Visual acuity did not improve beyond the preoperative acuity of light perception in both eyes. The lens remained clear, with adequate visualization of the posterior fundus..

\section{DISCUSSION}

Ocular injuries rank high among the causes of eye disorders seen in our environment. In one study ocular injuries was the third most common presentation at the general out-patient clinic in Ibadan, accounting for $12.8 \%$ of cases seen. ${ }^{10}$ In Lagos University Teaching Hospital, 104 patients were treated for ocular trauma between January and December $1986 .{ }^{11}$ In a study on childhood eye diseases in Ibadan, Ajaiyeoba noted that injury is the leading cause of eye diseases among children. ${ }^{12}$ One other report from the southeastern part of Nigeria has confirmed that trauma is a primary cause of uniocular blindness among young adults. ${ }^{13}$ At an outreach post at Lere in Kaduna State in northern Nigeria, the reported incidence of trauma is $3.4 \%{ }^{14}$

In the case reported in this paper, trauma was the major contributing factor to the eye disorder.

Our experience with the 2 eyes of this infant affirms the safety of lens sparing vitrectomy in the management of infantile vitreous haemorrhage as has been reported severally. ${ }^{1,4,5,15}$ Lens sparing vitrectomy has been popularized within the last decade in the interest of avoiding aphakic amblyopia in infants. ${ }^{6}$

This one-year old child represents the youngest patient ever to undergo a 3-port pars plana vitrectomy procedure in our retina service. The indication for this procedure is 
vitreous haemorrhage, secondary to an accidental trauma. The child was hit by a moving car, the point of impact being the right side of the head and face. There have been other studies reporting the occurrence of maxillofacial and other fractures in the paediatric age group, mostly caused by road traffic accidents. ${ }^{16,17}$ In a study by Ogunlewe et al. the incidence of facial fractures in the paediatric age group was reportedly higher than previously reported in Nigeria and the world over, with almost two-thirds of the cases occurring as a result of road traffic accidents. ${ }^{16}$

The incidence of ocular complications in the paediatric age group who have suffered facial trauma from road traffic accidents should be investigated, with particular reference to how quickly ophthalmologists are called in to see them.

Although the child in this case study regained all other neurological functions, her visual function remained impaired after vitrectomy. This underscores the importance of early assessment of the eyes following trauma to the head and facial region. This point should be stressed in the case of infantile or paediatric trauma, as trauma can be commonly overlooked in this group of patients who cannot express themselves very well.. Workers in the paediatric trauma and maxillofacial units should, therefore, be advised to call in ophthalmologists as soon as possible in the management of children with facial trauma. The patient should be examined even before consciousness is regained.

In this case, the patient had her initial ophthalmological review at about six weeks post trauma. It is possible that a steep prolonged rise in the post trauma intraocular pressure during the time of vitreous haemorrhaging resulted in compromising the optic nerve head perfusion, as well as the retinal vascular perfusion. Assuming therapy had been instituted to reverse this, damage and loss of vision could have been averted.

Common causes of infantile vitreous haemorrhage in the developed world include retinopathy of prematurity and trauma (mostly due to shaken-baby syndrome) and birth trauma. ${ }^{4}$

Vitreous haemorrhage caused by birth trauma and shaken baby syndrome may not be common in our environment. However, a pertinent question which should be investigated in developing countries, is, if birth trauma is the cause of vitreous haemorrhage, as is seen in the more developed world, does it include all forms of birth trauma to the head, including those that could arise from forceps delivery.

The outcome of pars plana vitrectomy in infants and paediatric age group has been good in selected cases of retinopathy of prematurity. ${ }^{15}$

In a reported series of eyes with dense vitreous haemorrhage caused by shaken baby syndrome, a poor visual prognosis was noted due to frequent concomitant retinal and visual cortical pathologic lesions. ${ }^{18}$ In another report it was stressed that visual outcome associated with shaken baby syndrome may be poor due to structural injury of the retina, optic nerve, or posterior visual pathways.$^{19} \mathrm{In}$ this report of shaken baby syndrome, 9 out of the 16 eyes had optic disc pallor or optic atrophy. ${ }^{19}$

The findings in this second series is similar to our own experience. As we observed, although the vitreous haemorrhage was successfully removed without compromise to the lens, visual acuity remained poor due to structural damage to the optic nerve, the retina, and the macular. It is also possible that the posterior visual pathway, including the visual cortex may have been involved in the damage. If so, the mechanism for this damage needs to be investigated, since the child regained all other neurological functions. Electrophysiological tests including VEP (visual evoked potential) and EEG (electroencephalogram) could be helpful.

\section{CONCLUSION}

In conclusion, this case does confirm the safety and efficacy of pars plana vitrectomy without removal of the crystalline lens in an infant for the treatment of a dense vitreous haemorrhage. It however points out that unlike the good visual outcome seen in some cases of retinopathy of prematurity and vitreous opacity due to causes other than trauma, the visual outcome following vitrectomy for vitreous haemorrhage secondary to trauma may be poor as a result of concomitant traumatic damage to the macular, optic nerve and other posterior visual pathway. This case also shows the danger in delaying proper ophthalmological examination in paediatric patients and makes a case for a very early assessment by the ophthalmologist of children with facial trauma / head injury with possibility of ocular involvement.

\section{REFERENCES}

1. Ferrone PJ, de Juan E Jr. Vitreous haemorrhage in infants . Arch. Ophthalmol 1994; 112 (9): 1185-9.

2. Mauire AM, Trese MT. Lens Sparing vitreoretinal surgery in infants. Arch Ophthalmol 1992; 110 (2): 284-6.

3. Capone A Jr, Trese MT. Lens sparing vitreous surgery for tractional stage 4: A retinopathy of prematurity retinal detachments. Ophthalmology 2001; 108 (11): 2068-70.

4. Luna JD, Caribaux LJ, Reviglio VE, Juarez CP. Lens-sparing surgery for retinopathy of prematurity. Ophthalmology 2003; 110(8): 1669-73.

5. Ferrone PJ, Harrison C, Trese MT. Lens clarity after lens -sparing vitrectomy in a paediatric population. Ophthalmology 1997; 104 (2): 273-8.

6. Mohney BG. Axial myopia associated with dense vitreous hemorrhage of the neonate. JAAPOS 2002; 6:348-353. 
7. Enock ME, Omoti AE, Alikah AA. Risk factors identification for ocular trauma in patients who presented in a sub urban tertiary care hospital in Nigeria. Saudi Med J 2007; 28(9): 13858.

8. Nzeh DA, Owoeye JF, Ademola-Popoola DS, Uyanne I. Sonographic evaluation of ocular trauma in Ilorin, Nigeria. Eur J Ophthalmol 2006; 16(3):453-7.

9. Ukponmwan CU, Marchien TT. Ultrasonic diagnosis of orbito-ocular diseases in Benin City, Nigeria. Niger Postgrad Med J. 2001; 8(3):123-6.

10. Scot SC, Ajaiyeoba AI. Eye diseases in a general out patient clinic in Ibadan. Niger J. Med 2003; 12(2): 76-80.

11. Adefule-Ositelu AO, Soetan II, Akinsola FB. Ocular trauma in Lagos. West Afr J. Med 1996; 15(4): 210 - 4.

12. Ajaiyeoba A. Childhood eye diseases in Ibadan. Afr J. Med Sci. 1994; 23(3): 227-31.

13. Nwosu SN. Ocular problems of young adults in rural Nigeria. Int Ophthalmol 1998; 22(5): 259-63.
14. Ogwurike SC. Ocular disease at Lere Local Government outreach post in Kaduna State of northern Nigeria. West Afr J Med 2007; 26(1): 20-3.

15. Moshfeghi AA, Awner S, Salam GA, Ferrone PJ . Excellent visual outcome and reversal of dragging after lens sparing vitrectomy for progressive tractional stage 4: A retinopathy of prematurity retinal detachment. Retina 2004; 24(4): 615-6.

16. Ogunlewe MO, James O, Ladeinde AL, Adeyemo WL. Pattern of paediatric maxillofacial fractures in Lagos, Nigeria. Int $J$ Paediatr Dent 2006;16(5): 358-62 .

17. Nwadinigwe CU, Ihezie CO, Iyidiobi EC. Fractures in children. Niger J. Med 2006; 15(1): 81-4.

18. Antonio C. Lens-sparing vitreous surgery for infantile amblyogenic vitreous hemorrhage. Retina 2003; 6: 792 - 5 .

19. Matthews GP, Das A. Dense vitreous hemorrhage predicts poor visual and neurological prognosis in infants with shaken baby syndrome. J Pediatr Ophthalmol Strabismus 1996; 33: 260265. 